\title{
Analysis of deformation characteristics and microstructure variation of thick plate during asymmetric shear rolling
}

\author{
Tao Zhang ${ }^{1, *}$, Lei $\mathrm{Li}^{1}$, and Shihong $\mathrm{Lu}^{1}$ \\ ${ }^{1}$ College of Mechanic and Electrical Engineering, Nanjing University of Aeronautics and Astronautics, 210016 Nanjing, China
}

\begin{abstract}
Aluminium alloy thick plate is widely used in field of aerospace and automobile industry for its light weight, high strength, good corrosion resistance and formability. Hot rolling is the key process of preparation for thick plates, however, the conventional symmetrical rolling process causes large deformation on the surface and small deformation in the central portion of the thick plate, resulting in inhomogeneous deformation, microstructure and property distributions through the thickness direction of the plate. Asynchronous rolling is beneficial for deformation permeation into the central part of thick plate with introduction of strong shear strain due to velocity asymmetry of two work rolls. However, the plate will bend towards the slower roll due to deformation asymmetry at upper and lower surface of the plate and serious bending is bad for multi-pass rolling process. Asymmetrical shear rolling with velocity asymmetry and geometry asymmetry is adopted in this study. Compared to asynchronous rolling, there is a horizontal offset distance of upper roll in outlet direction. A moment will be applied to the plate in the opposite direction of bending towards the slower roll by an offset distance of the slower roll. The comparisons of distributions of equivalent strain, temperature, DRX fraction and average grain size between symmetrical rolling and asymmetrical shear rolling are studied. The effects of velocity asymmetry and offset distance on microstructure variation and microstructure distribution at different positions of the plate are studied. Due to larger velocity of lower work roll, larger equivalent strain and higher temperature appear at lower layer of the plate, which results in higher DRX fraction and finer grain size. The speed ratio contributes greatly to sufficient DRX process and finer grain size; while the ascending offset distance decreases the DRX fraction. Therefore, speed ratio plays significant role in deformation permeation and grain refinement in asymmetrical shear rolling and the offset distance is mainly used to decrease the bending behaviour of the plate. This study can provide guidance to thick plate preparation with good properties in asymmetrical rolling process.
\end{abstract}

Keywords: Rolling、Deformation、 Microstructure

\section{Introduction}

With the development of aerospace industry, aluminum alloy thick plates with large thickness and high performance (even to $250 \mathrm{~mm}$ and $700 \mathrm{MPa}$ ) are in great need. Hot rolling is the main process for thick plate preparation. However, large deformation is mainly generated on the surface of the plate in symmetrical rolling and the properties at the centre are poorer than that on the surface, which cause severe property inhomogeneities. Differential speed rolling is useful for deformation permeation into the centre of the thick plate by inducing strong shear strain. However, there is severe bending behaviour of the plate as the deformation between upper and lower surface of the plate is uneven, which may cause accidents in production process. Therefore, a new method of asymmetrical shear rolling is adopted to solve this problem [1]. The bending behaviour can be effectively controlled with the basis of obtainment of strong shear deformation.

Finite element model (FEM) was widely used to study the deformation distribution [2] and temperature variation [3-4] during asymmetrical hot rolling. Serajzadeh [5] studied the strain inhomogeneity in asymmetrical hot strip rolling through FEM simulation. Zuo [6] analyzed the shear strain quantificationally of pure $\mathrm{Al}$ in asymmetric rolling by experiments and observed the grain refinement after strong shear deformation. Ding [7] studied the temperature distribution of $\mathrm{Mg}$ alloy and the temperature variation at different positions of the plate in the hot rolling by experiment and FEM. In hot rolling process, there are different deformation mechanisms, such as work hardening (WH), dynamic recovery (DR) and dynamic recrystallization (DRX) [8]. Yin [9] studied the mechanism of continuous DRX during hot compressive deformation by experiments. Lin [10] establishend a

Corresponding author: 297zhangtao@nuaa.edu.cn 
numerical model to simulate the microstructure evolution in a three-dimensional hot upsetting process and conducted experiments to verigy the simulation results. Cellular automata (CA) method is a new method to simulate the microstructure variation due to DRX and it can be used to obtain the microstructure topology [11]. Zheng [12] used CA model to simulate the austenite recrystallization during multi-pass steel strip hot rolling and established a model to predict the microstructure variation. $\mathrm{Li}$ [13] studied the relationship between mechanical properties and microstructure of AA1060 aluminum plate during snake rolling.

However, the comparisons of strain and temperature between symmetrical rolling and asymmetrical shear rolling were rarely published as there are velocity asymmetry and geometry asymmetry in asymmetrical shear rolling. Most studies focused on the effect of speed ratio on deformation distribution of the plate. Furthermore, the effects of speed ratio and offset distance on microstructure evolution in asymmetrical shear rolling need to be further studied. In this study, coupled numerical models are established to compare the distributions of equivalent strain, temperature, DRX fraction and average grain size between symmetrical rolling and asymmetrical shear rolling. CA models are established to simulate the microstructure topology and grain variation at different parameters due to DRX process.

\section{Asymmetrical shear rolling}

Fig.1 shows the diagrams of symmetrical rolling and asymmetrical shear rolling. In symmetrical rolling, the deformation zone is divided into backward slip zone (BSZ) and forward slip zone (FSZ) as the velocities of two work rolls are the same. However, in asymmetrical shear rolling, the velocity of lower work roll is larger than that of upper lower roll and there is an offset distance $(S)$ in outlet direction of two rolls. Therefore, a new zone of cross shear, the so called cross shear zone (CSZ) appears as the neutral points of the upper and lower surface are not in the same verticle position. In $\mathrm{CSZ}$, the frictional direction is the same at the upper and lower surface, which is different from that in BSZ and FSZ. As a result, the plate is subjected to compressive deformation and strong shear deformation in CSZ, which is beneficial for deformation permeation of thick plate. The plate will bend up for the smaller velocity of upper roll. In asymmetrical shear rolling, the offset distance of the upper roll will bring a moment in the oppsite direction of bending up, which is useful to decrease the bending behaviour of the plate.
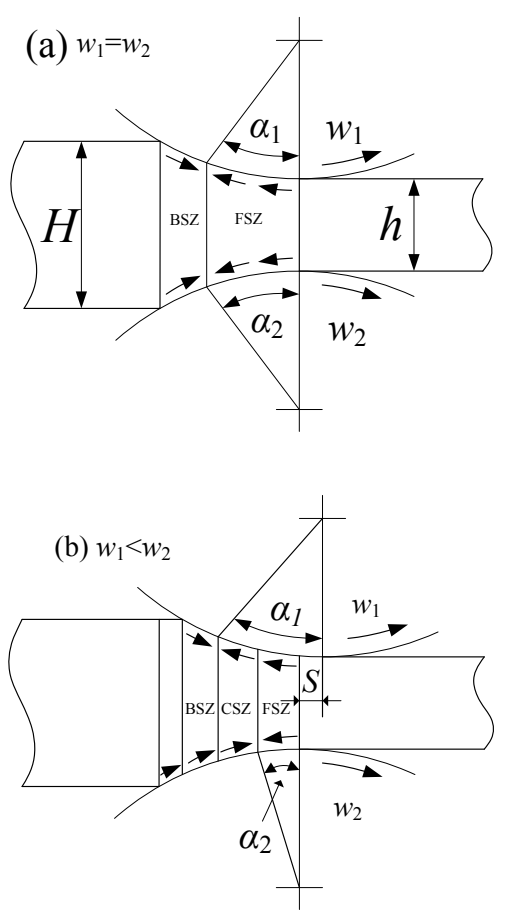

Fig. 1. Schematic of different rolling methods: (a) symmetrical rolling (b) asymmetrical shear rolling

\section{Model descriptions}

\subsection{Coupled numerical model}

In order to simulate the deformation distribution of the plate in symmetrical rolling and asymmetrical shear rolling, single pass rolling FEM models are established. The work rolls are defined as rigid bodies and the plate is 7055 aluminum alloy and its material constants and constitutive equation are shown in author's previous article [14]. The rolling parameters of asymmetrical shear rolling are shown in Table 1; while the speed ratio is set 1 and offset distance is set 0 in symmetrical rolling.

In hot rolling, DRX occurs when the true strain reaches the critical value and DRX plays a vital role in grain refinement as new dynamic recrystallized grains are formed at grain boundary of the matrix. DRX fraction, recrystallized grain size and average grain size were widely used to describe the DRX behaviour by Sellars and Yada [15-16], as shown in Eqs. (1)-(6).

$$
\begin{gathered}
\varepsilon_{p}=1.697 \times 10^{-5} \dot{\varepsilon}^{0.15975} \exp \left(\frac{42448}{R T}\right) \\
\varepsilon_{c}=0.8 \varepsilon_{p} \\
X_{d}=1-\exp \left[-3.4 \times 10^{-2}\left(\frac{\varepsilon-\varepsilon_{c}}{\varepsilon_{0.5}}\right)^{1.065}\right]
\end{gathered}
$$




$$
\begin{gathered}
\varepsilon_{0.5}=7.57 \times 10^{-4} \dot{\varepsilon}^{0.09349} \exp \left(\frac{25707}{R T}\right) \\
D_{d}=1.0473 \times 10^{3} \dot{\varepsilon}^{0.129} \exp \left(\frac{-21542}{R T}\right) \\
\bar{D}=X_{d} D_{d}+\left(1-X_{d}\right) D_{0}
\end{gathered}
$$

Where $\varepsilon_{\mathrm{p}}$ is peak strain, $\varepsilon_{\mathrm{c}}$ is critical strain, $X_{\mathrm{d}}$ is DRX fraction, $\varepsilon_{0.5}$ is the strain corresponding to $50 \% \mathrm{DRX}$ fraction, $D_{d}$ is recrystallized grain size, $D_{0}$ is initial grain size, $\bar{D}$ is average grain size after deformation.

Table 1. Single rolling parameters

\begin{tabular}{|c|c|}
\hline Parameter & Value \\
\hline Initial rolling temperature, $T /{ }^{\circ} \mathrm{C}$ & 410 \\
\hline Work roll radius, $R / \mathrm{mm}$ & 500 \\
\hline Shear friction coefficient, $m$ & 0.4 \\
\hline Velocity of lower roll, $\omega_{2} /(\mathrm{rad} / \mathrm{s})$ & $2 \sim 2.6$ \\
\hline Velocity of upper roll, $\omega_{1} /(\mathrm{rad} / \mathrm{s})$ & 2 \\
\hline Initial thickness, $H / \mathrm{mm}$ & 250 \\
\hline Pass reduction, $\Delta h / \mathrm{mm}$ & 30,50 \\
\hline Speed ratio, $i=\omega_{2} / \omega_{1}$ & $1.00 \sim 1.3$ \\
\hline Offset distance, $S / \mathrm{mm}^{2}$ & $0 \sim 70$ \\
\hline $\begin{array}{c}\text { Heat transfer of convection and } \\
\text { radiation, } h 1 /\left(\mathrm{W} \cdot \mathrm{m}^{2} \cdot \mathrm{K}^{-1}\right)\end{array}$ & 5 \\
\hline $\begin{array}{c}\text { Heat transfer of contact and cooling, } h_{2} \\
/\left(\mathrm{W} \cdot \mathrm{m}^{2} \cdot \mathrm{K}^{-1}\right)\end{array}$ & 30000 \\
\hline
\end{tabular}

\subsection{Cellular automata model}

CA model coupled with mathematical random algorithm and physical metallurgy theory is used to simulate the microstructure evolution. The plate is divided into cells discreted in time and space and rules can be added to simulate the cell change in each CA step. There are several parameters in each cell, such as crystal orientation, dislocation density, temperature, strain rate and recrystallized flag.

The dislocation density increases with strain by WH and decreases at high temperature by DR and DRX, which can be described by Kocks-Meching (KM) model [17] in Eq. (7). When the dislocation density reaches a critical value, new dynamic recrystallized nuclei appear at grain boundary. The nucleation rate can be described by Ding and Guo model [18], as shown in Eq. (8). After the nucleation, the nuclei will grow into recrystallized grains with certain sizes and the dislocation density difference between recrystallized grains and matrix provides the driving force for the growth. The grain growth velocity is calculated by Eqs. (9)-(10).

$$
\begin{gathered}
\frac{d \rho}{d \varepsilon}=k_{1} \sqrt{\rho}-k_{2} \rho \\
\dot{n}=C \dot{\varepsilon}^{m} \exp \left(\frac{-Q_{a c t}}{R T}\right) \\
v=M p \\
M=\frac{b}{k T} \lambda D_{\mathrm{b}} \exp \left(\frac{-Q_{\mathrm{b}}}{R T}\right)
\end{gathered}
$$

Where $\rho$ is the dislocation density, $k_{1}$ and $k_{2}$ are work hardening and dynamic softening coefficient, respectively; $\dot{n}$ is nucleation rate, $m$ is the sensitivity coefficient of strain rate, $Q_{\text {act }}$ is active energy, $\mathrm{T}$ is temperature, $v$ is grain growth velocity, $M$ is grain boundary (GB) mobility, $p$ is pressure, $b$ is the modulus of Burger's vector, $k$ is Boltzmann constant, $\lambda$ is the thickness of characteristic GB, $D_{\mathrm{b}}$ is the boundary selfdiffusion coefficient.

\section{Results and discussion}

\subsection{Comparisons of two rolling technologies}

In asymmetrical shear rolling, the speed ratio and offset distance will change the deformation zones, and then change the deformation and temperature distribution of the plate. Fig.2 shows the comparison of equivalent strain and temperature of two rolling ways. The equivalent strain distribution is symmetrical and it is near to " $\mathrm{M}$ " shape in symmetrical rolling. Due to the contact friction force, the strain at the surface is smaller than that at sub-surface (nearly 1/8 from surface). However, the equivalent strain at lower layer of the plate is much larger than that at upper layer and the strain distribution is obvious asymmetrical in asymmetrical shear rolling. The metal flow velocity at lower layer is larger as the lower roll has a faster velocity. In asymmetrical shear rolling, the maximum strain at lower layer is $15.1 \%$ larger than that in symmetrical rolling, while the strain at upper layer is $8.9 \%$ smaller. Due to strong shear strain in CSR, the equivalent strain in asymmetrical shear rolling is larger than in symmetrical rolling, which illustrates that speed ratio is beneficial for deformation permeation. The temperature distribution is also asymmetrical and the temperature at lower layer is higher than the upper layer. The temperatures at both surfaces decrease due to heat exchange with environment and work rolls. The temperature variation is related to plastic deformation heat and heat exchange, especially for the contact heat exchange. In asymmetrical shear rolling, the heat generated by plastic deformation at lower layer is larger than in symmetrical rolling; meanwhile, the contact time is shorter for the larger velocity of lower roll. More plastic deformation heat and 
less heat exchange results in a higher temperature at lower layer.

Large strain and high temperature are beneficial to DRX process and grain refinement in hot rolling. Fig.3 shows the comparison of DRX fraction and average grain size of two rolling ways. The DRX fraction distribution is similar to that of equivalent strain. The DRX fraction at lower sub-surface is $32 \%$ and it is much larger than upper sub-surface of $25 \%$ due to its large deformation and high temperature. As a result, the average grain size reduces from $100 \mu \mathrm{m}$ (initial value) to $80.4 \mu \mathrm{m}$ at lower sub-surface. As for the centre point of the plate, the DRX fraction increase and the average grain size decreases slightly in asymmetrical shear rolling.
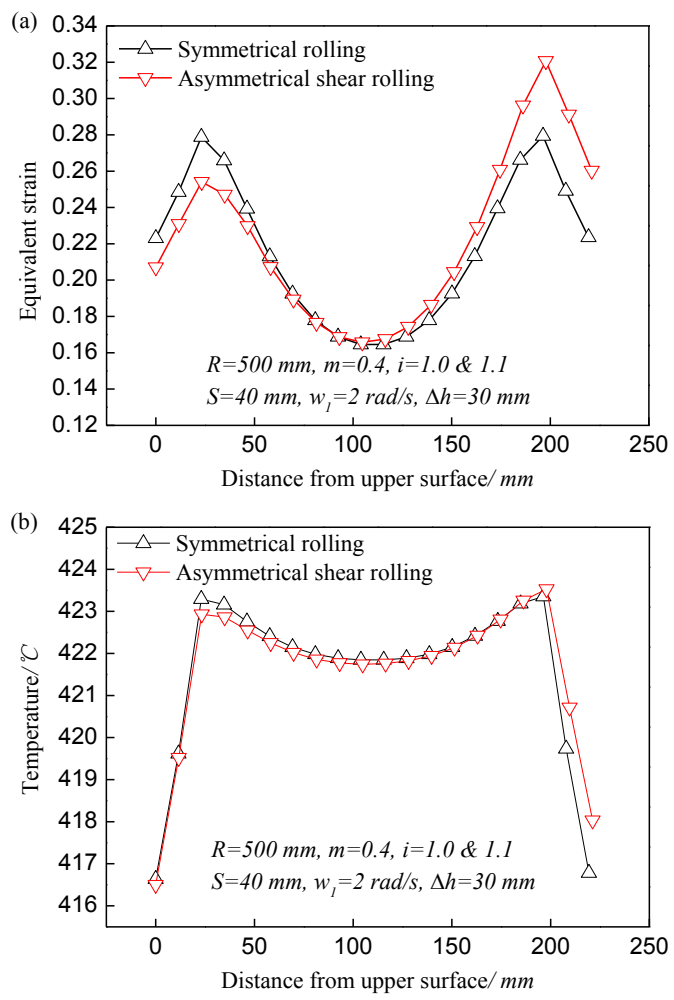

Fig. 2. Comparisons of (a) equivalent strain (b) temperature between two rolling ways

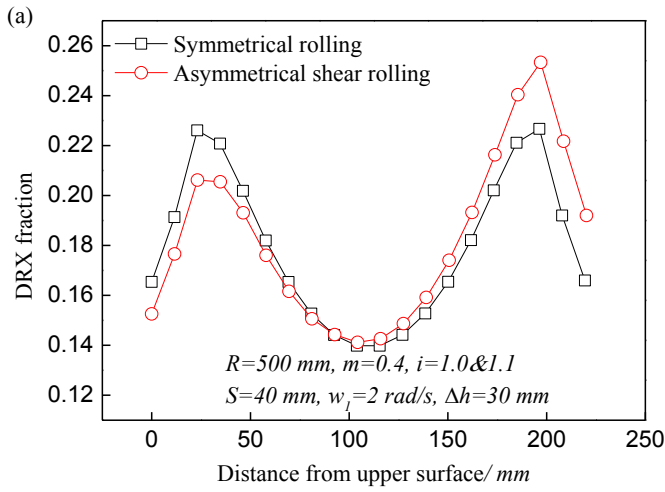

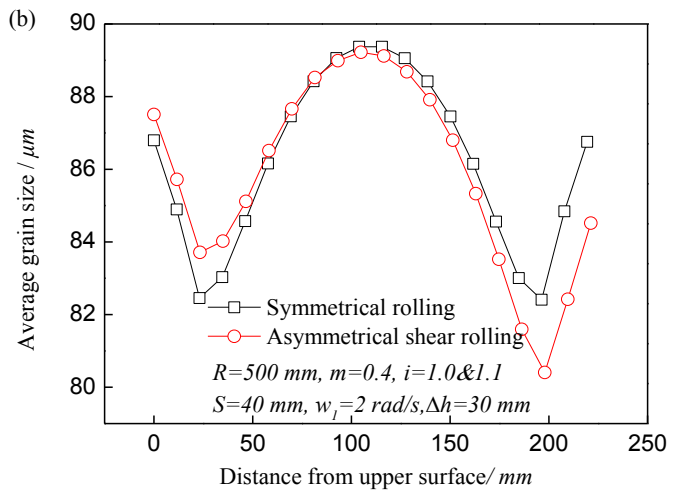

Fig. 3. Comparisons of (a) DRX fraction (b) average grain size between two rolling ways

\subsection{Microstructure visualization}

The microstructure visualization can be achieved in CA models and Fig. 4 shows CA simulated microstructure evolution at different strains under temperature of $450{ }^{\circ} \mathrm{C}$ and strain rate of $0.01 \mathrm{~s}^{-1}$. The white region represents matrix and the colored region is recrystallized grain, different colors represent grains with different orientations. With increase of strain, the nuclei start to appear at grain boundary for its high energy and then grow into recrystallized grains with a certain size. Finally, the recrystallized grains grow into a stable size and will not grow with further increase of strain. The deformation energy inside the material increases sharply with ascending strain, which provides sufficient driving force for the growth. The nuclei grow into recrystallized grains by absorbing grains of the matrix. The dislocation densities of the new recrystallized grains and the matrix both increase with ascending strain. When they reach the saturation values, the dislocation density difference between the matrix and recrystallized grains will decrease to zero, in which the new grains will stop growing up. It is obvious that the DRX fraction increases with ascending strain, as a result, the grains are significantly refined.

Fig.5 shows microstructure variation at different deformation zones at center point of plate under $i=1.1$ and $S=40 \mathrm{~mm}$ in asymmetrical shear rolling. It is obvious that the strain increases from BSZ to FSZ, which can be seen from the shape of the matrix grains. A few nuclei start to appear under small deformation in BSZ because the plate is just bited into the deformation zone. The grains are almost equiaxed in BSZ. The DRX fraction and the recrystallized grain size increase distinctly in CSR. The strong shear deformation contributes greatly to strain enlargement; meanwhile, the temperature rises quickly due to large plastic deformation heat, which obviously decrease the critical strain for DRX and enhances thermal motion of molecules and atoms inside the material. The DRX fraction is $1.8 \%$ and the average grain size is $100 \mu \mathrm{m}$ in Fig.5(a), while they change to $10 \%$ and $93.2 \mu \mathrm{m}$ in Fig.5(b). In FSZ, there is a slight increase of DRX fraction and then the grain can be further refined. 


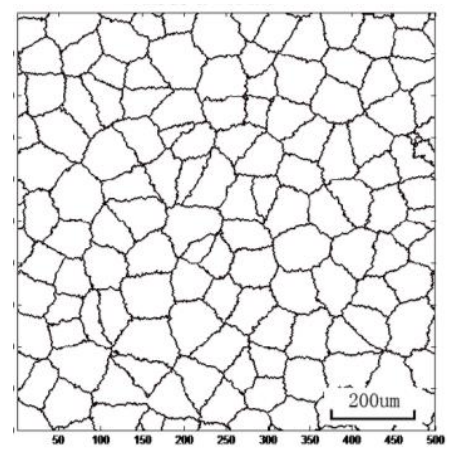

(a) $\varepsilon=0$

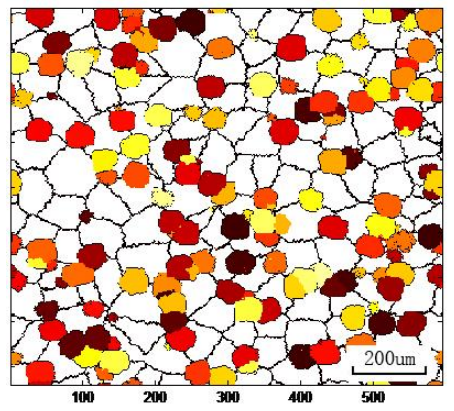

(b) $\varepsilon=0.19$

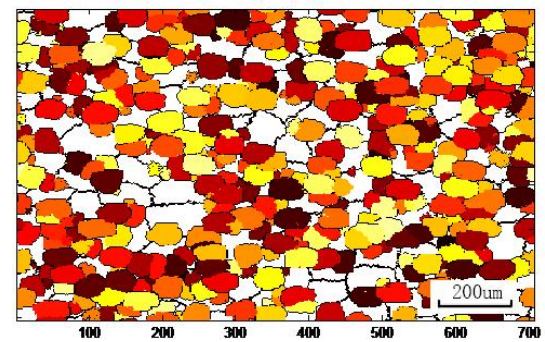

(c) $\varepsilon=0.35$

Fig. 4. Microstructure evolution at different strains under temperature of $450{ }^{\circ} \mathrm{C}$ and strain rate of $0.01 \mathrm{~s}^{-1}$

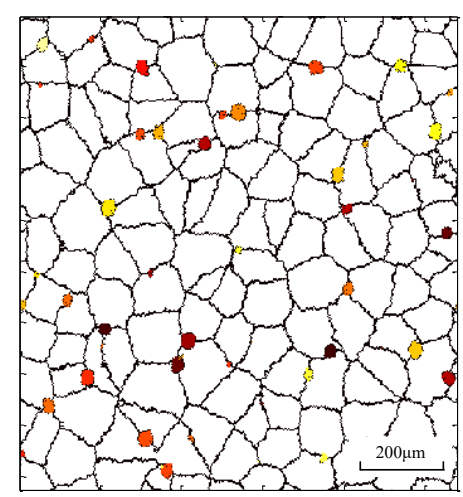

(a) backward slip zone

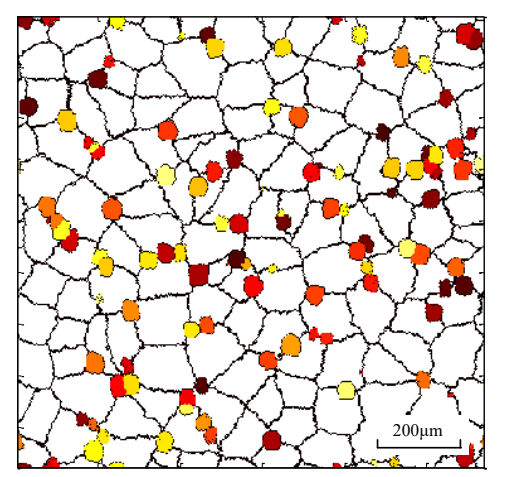

(b) cross shear zone

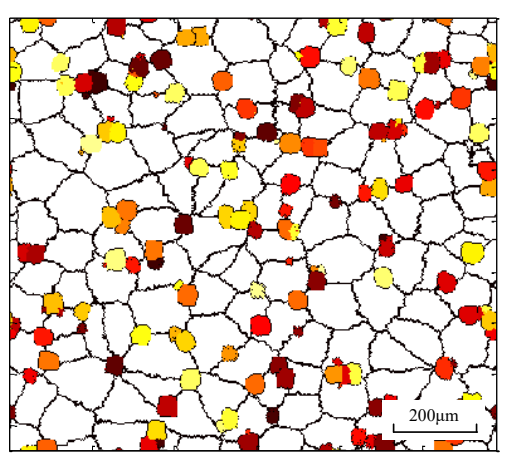

(c) forward slip zone

Fig. 5. Microstructure variation at different deformation zones at center point of plate under $i=1.1$ and $S=40 \mathrm{~mm}$

The speed ratio and offset distance are important parameters in asymmetrical rolling. Fig. 6 shows the effect of speed ratio on DRX fraction and average grain size. The length of CSR and shear strain increase dramatically with ascending speed ratio [19]. The DRX fraction increases sharply and the average grain size decreases when the speed ratio changes from 1.0 to 1.2. Due to increase of velocity difference, the equivalent strain and temperature both increase obviously, which provide more deformation energy for DRX process and reduce the critical strain for occurence of DRX. As recrystallized grain size is much smaller than initial grain size, the increasing DRX fraction results in severe grain refinement. However, the equivalent strain almost keeps

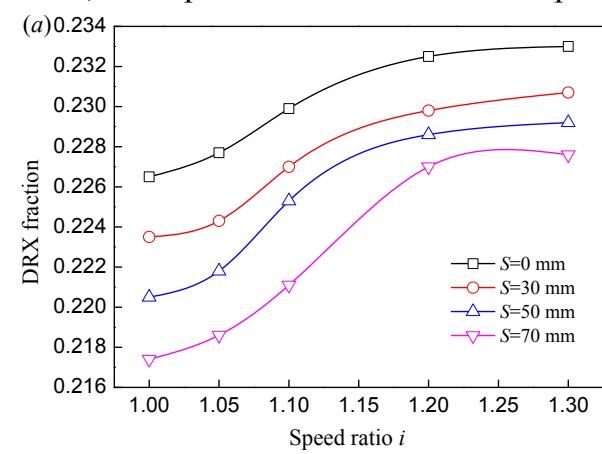

Fig. 6. Effect of speed ratio on DRX fraction and average grain size at center point of plate steady by further increase of speed ratio from 1.2 to 1.3 [20]. Therefore, there are slight variations of DRX fraction and average grain size.

The effect of offset distance on DRX fraction and average grain size is shown in Fig.7. The DRX fraction decreases and average grain size increases with ascending offset distance. For a given pass reduction, the existence of offset distance will decrease the actual rolling reduction, which reduces the equivalent strain of the plate. Therefore, the offset distance is mainly used to decrease the bending behavior of the plate during asymmetrical shear rolling and it should be properly adjusted according to the value of speed ratio.

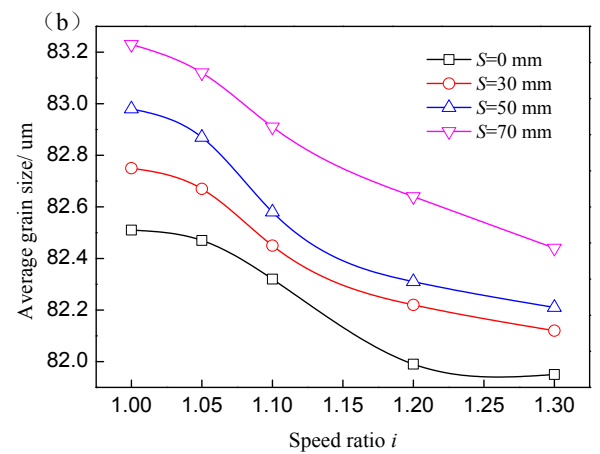



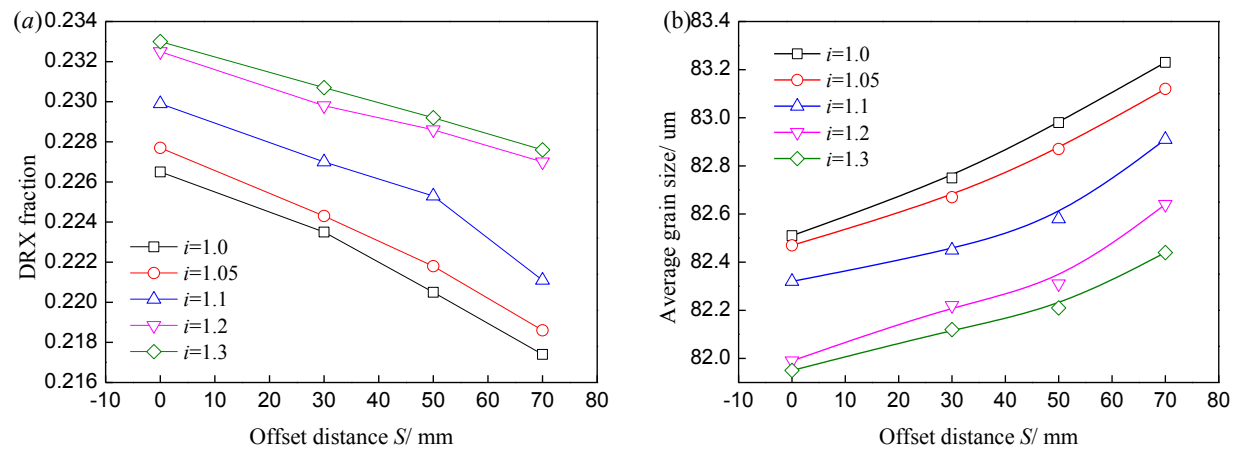

Fig. 7. Effect of offset distance on DRX fraction and average grain size at center point of plate

\section{Conclusions}

Coupled numerical models are established to compare the distributions of equivalent strain, temperature, DRX fraction and average grain size between symmetrical rolling and asymmetrical shear rolling. CA models are established to simulate the microstructure topology variation of the plate due to DRX process in hot rolling.

1. The distributions of equivalent strain, temperature are asymmetric in asymmetrical shear rolling. The strain and temperature at lower layer of the plate is higher due to faster velocity of lower work roll.

2. More sufficient DRX and finer grain appear at lower layer of the plate; meanwhile, the grain size at the centre is smaller in single asymmetrical shear rolling, which illustrates that asymmetrical shear rolling is potential for grain refinement.

3. Increasing speed ratio is beneficial to enlarge DRX fraction and decrease average grain size; while the ascending offset distance reduce the DRX extent. Therefore, speed ratio is the vital factor for grain refinement and offset distance is mainly used for bending control of the plate.

Acknowledgments: This project is supported by National Natural Science Foundation of China (Grant No.51705248); Natural Science Foundation of Jiangsu pvovince, China (Grant No.BK20170785) and Scientific Research Staring Foundation for Talent Introduction of Nanjing University of Aeronautics and Astronautics (Grant No. 90YAH17038)

\section{References}

1. Y. Fu, S.S. Xie, B.Q. Xiong, G.J. Huang, L. Cheng, J. Wuhan. Univ. Technol. 27(02): 247-251 (2012).

2. S. Serajzadeh, A. K. Taheri, M. Nejati, J. Izadi, M. Fattahi, J. Mater. Process. Tech. 128(1): 88-99 (2002).

3. M. Bagheripoor, H. Bisadi, Appl. Therm. Eng. 31(10): 1556-1565 (2011).

4. R. B. Mei, C. S. Li, X. H. Liu, B. Han, J. Iron. Steel. Res. Int. 17(2): 17-21 (2010).
5. H. Yang, M. Wang, L.G. Guo, Z.C. Sun, Comp. Mater. Sci. 44(2): 611-621(2008).

6. F.Q. Zuo, J.H. Jiang, A.D. Shan, J.M. Fang, X.Y. Zhang, T. Nonferr. Metal. Soc. 18(4): 774-777 (2008).

7. Y. Ding, Q. Zhu, Q. Le, Z. Zhang, L. Bao, J. Cui, J. Mater. Process. Tech. 225: 286-294 (2015).

8. C. Shi, J. Lai, X.G. Chen, Materials. 7(1), 244 (2014).

9. X. Q. Yin, C. H. Park, Y. F. Li, J. Alloy. Compd. 693:426-431(2017).

10. Y. C. Lin; M. S. Chen, J. Mater. Process. Tech. 209(9):4578-4583 (2009).

11. M. Sitko, M. Pietrzyk, L. Madej, J. Comput. Sci. 16: 98-113 (2016).

12. C. Zheng, N. Xiao, D. Li, Comp. Mater. Sci. 44(2), 507-514 (2008).

13. S. Li, N. Qin, J. Liu, Mater. Design. 90: 1010-1017 (2016).

14. T. Zhang, Y. X. Wu, H. Gong, Mechanika. 22(5):359-365 (2016).

15. C. M. Sellars, J. A. Whiteman, Met. Sci. 13(34):187-194 (1979).

16. T. Senuma, H. Yada, H. Yoshimura, Adv. Mater. Res. 626(6):430-435 (1986).

17. Y. Liu, Y. Lin, H. Li, D. Wen, X. Chen, M. Chen, Mat. Sci. Eng. A-Struct. 626: $432-440$ (2015).

18. R. Ding, Z.X. Guo, Acta. Mater. 49(16): 3163-3175 (2001)

19. T. Zhang, Y. X. Wu, H. Gong, J. Cent. South Univ. 24(2):296-302 (2017).

20. Zhang T, Wu Y X, Gong H, J. Cent. South Univ. 22: 2463-2469 (2015) 